\title{
An Optimized Synthetic Process for the Substitution of Cobalt in Nickel- Rich Cathode Materials
}

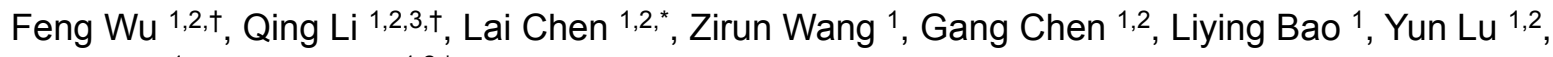 \\ Shi Chen ${ }^{1}$, Yuefeng Su ${ }^{1,2, *}$ \\ ${ }^{1}$ School of Materials Science and Engineering, Beijing Key Laboratory of Environmental Science and Engineering, \\ Beijing Institute of Technology, Beijing 100081, China. \\ ${ }^{2}$ Beijing Institute of Technology Chongqing Innovation Center, Chongqing 401120, China. \\ ${ }^{3}$ Energy Storage and Distributed Resources Division, Lawrence Berkeley National Laboratory, Berkeley, CA 94720, USA.
}

\begin{abstract}
High-performance rechargeable lithium ion batteries have been widely applied in electrochemical energy storage fields, such as, energy storage grids, portable electronic devices, and electric vehicles (EVs). However, the energy density of lithium ion batteries needs to be increased, and the

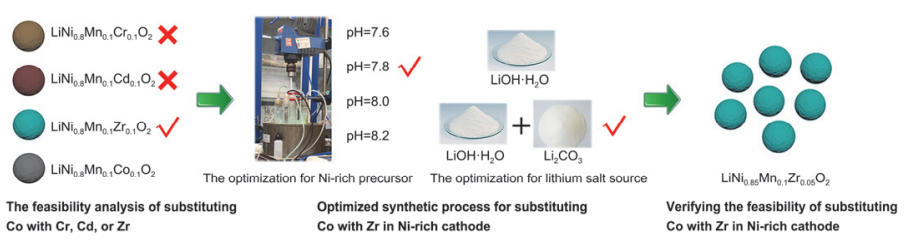
cost of battery materials could be further reduced for wider commercial applications. An Ni-rich cathode, $\mathrm{LiNi}_{x} \mathrm{Mn}_{y} \mathrm{Co}_{1-x-y} \mathrm{O}_{2}$ $(x>0.8)$, with high specific capacity is the most promising material for next-generation Li-ion batteries. $\mathrm{LiNi}_{x} \mathrm{Mn}_{y} \mathrm{Co}_{1-x-y} \mathrm{O}_{2}$ $(x>0.8)$ contains three transition metal elements, $\mathrm{Ni}, \mathrm{Mn}$, and $\mathrm{Co}$, respectively. The role of $\mathrm{Ni}^{2+}$ is to provide high capacity for recharge The role of $\mathrm{Mn}^{4+}$ is to stabilize the lattice structure during charging-discharging cycling. Crucially, the role of $\mathrm{Co}^{3+}$ in Ni-rich materials is to improve the electrical conductivity and inhibit cation disorder in the lattice during electrochemical cycling. However, $\mathrm{Co}$ is both in shortage and expensive, which limits its worldwide commercial application. This work investigates substituting Co with other abundant and cheap transition metals. Transition metal ions $\mathrm{Cr}^{3+}, \mathrm{Cd}^{2+}$, and $\mathrm{Zr}^{4+}$ can replace $\mathrm{Co}^{3+}$ in Ni-rich cathode materials. $\mathrm{LiNi} 0.8 \mathrm{Cr}_{0.1} \mathrm{Mn}_{0.1} \mathrm{O}_{2}$, LiNio.8 $\mathrm{Cd}_{0.1} \mathrm{Mn}_{0.1} \mathrm{O}_{2}$, and LiNio.8 $\mathrm{Zr}_{0.1} \mathrm{Mnn}_{0.1} \mathrm{O}_{2}$ were synthesized by a co-precipitation method. $\mathrm{Zr}$ was found to be the best candidate for replacing Co in Ni-rich cathode materials. This study investigated $\mathrm{Zr}^{4+}$-doped Co-free Ni-rich materials. Initially, a carbonate co-precipitation process was used to synthesize $\mathrm{Ni}_{0.8} \mathrm{Zr}_{0.1} \mathrm{Mn}_{0.1} \mathrm{CO}_{3}$. This is due to that $\mathrm{Zr}^{3+} / \mathrm{Zr}^{4+}$ ions are not precipitated in the strong alkali solution, and the $\mathrm{pH}$ during hydroxide co-precipitation and carbonate co-precipitation processes are approximately 11 and 8 , respectively. Therefore, the carbonate co-precipitation synthesis method was chosen. $\mathrm{Ni}_{0.8} \mathrm{Zr}_{0.1} \mathrm{Mn}_{0.1} \mathrm{CO}_{3}$ was synthesized by carbonate co-precipitation at $\mathrm{pH}=7.6,7.8,8.0$, and 8.2. After electrochemical analysis, $\mathrm{pH}=7.8$ was identified as the optimal value. The next stage of the research involved completing an electrochemical performance comparison on two lithium sources. The following lithium sources were added to the precursor; $\mathrm{LiOH} \cdot \mathrm{H}_{2} \mathrm{O}$, and a $1: 1$ mixture of $\mathrm{LiOH} \cdot \mathrm{H}_{2} \mathrm{O}$ and $\mathrm{Li} 2 \mathrm{CO}_{3}$. The lithium source with the $1: 1$ mixture, exhibited better performance for the Ni-rich cathode, $\mathrm{LiNi}_{0.8} \mathrm{Zr}_{0.1} \mathrm{Mn}_{0.1} \mathrm{O}_{2}$. In this study, the ideal doping amount of $\mathrm{Zr}$ in Ni-rich materials was 0.05 . In conclusion, by careful control of co-precipitation $\mathrm{pH}$ and $\mathrm{Li}$ source, the $\mathrm{Zr}$ doped cobalt free Ni-rich cathode $\mathrm{LiNi}_{0.85} \mathrm{Mn}_{0.1} \mathrm{Zr}_{0.05} \mathrm{O}_{2}$ delivered a discharge capacity of $179.9 \mathrm{mAh} \cdot \mathrm{g}^{-1}$ at $0.2 \mathrm{C}$. This was achieved between the voltage range of $2.75-4.3 \mathrm{~V}$, with an 80 cycle capacity retention of $96.52 \%$.
\end{abstract}

Key Words: Lithium ion battery; Nickle-rich cathode material; Cobalt free; Cycling stability

Received: July 6, 2020; Revised: August 7, 2020; Accepted: August 18, 2020; Published online: August 24, 2020.

† These authors contribute equally to this work.

*Corresponding authors. Emails: chenlai144@sina.com (L.C.); suyuefeng@bit.edu.cn (Y.S.).

The project was supported by the National Key R\&D Program of China (2016YFB0100301), the National Natural Science Foundation of China (21875022, 51802020, U1664255), the Science and Technology Innovation Foundation of Beijing Institute of Technology Chongqing Innovation Center (2020CX5100006), the Beijing Institute of Technology Research Fund Program for Young Scholars, and the Young Elite Scientists Sponsorship Program by CAST (2018QNRC001). 国家重点研发项目(2016YFB0100301), 国家自然科学基金(21875022, 51802020, U1664255), 北京理工大学重庆创新中心科技创新计划项目 (2020CX5100006), 北京理工大学 “青年教师学术启动计划” 项目和中国科学技术协会青年人才托举计划(2018QNRC001)资助

(C) Editorial office of Acta Physico-Chimica Sinica 


\title{
高镍正极材料中钴元素的替代方案及其合成工艺优化
}

\author{
吴锋 $1,2,+$, 李晴 $1,2,3,+$, 陈来 $1,2,{ }^{*}$, 王紫润 ${ }^{1}$, 陈刚 1,2 , 包丽颖 ${ }^{1}$, 卢债 1,2 , 陈实 ${ }^{1}$, \\ 苏岳锋 $1,2,{ }^{*}$ \\ 1 北京理工大学材料学院, 环境科学与工程北京市重点实验室, 北京 100081 \\ 2 北京理工大学重庆创新中心, 重庆 401120 \\ 3 劳伦斯伯克利国家实验室能量存储与分布式资源部门，美国加州 94720
}

\begin{abstract}
摘要: 高镍三元正极材料 $\mathrm{LiNi}_{x} \mathrm{Mn}_{y} \mathrm{Co}_{1-x-y} \mathrm{O}_{2}(x>0.8)$ 因其高能量密度而备受瞩目。在高镍三元正极材料中, Co 不但有助于 增强层状正极材料结构稳定性, 而且能够提高正极材料导电性能, 因此被认为是一种非常重要的元素。但是由于目前全球 范围内钴矿资源紧缺, 在一定程度上限制了含钴正极材料在新能源电动汽车领域的发展应用。基于此, 本文将不同的过渡 金属离子掺杂到高镍层状材料中形成无钴化正极材料, 并进行高镍正极材料无钴化的可行性分析。通过实验对比发现, 资源 存储量丰富并且价格低廉的 $\mathrm{Zr}$ 在一定程度上可以取代 Co 元素, 得到的正极材料 $\mathrm{LiNio} .85 \mathrm{Mn} 0.1 \mathrm{Zr} 0.05 \mathrm{O}_{2}$ 表现出良好的电化学 性能, 在 $0.2 \mathrm{C}$ 倍率以及 2.75-4.3 V 的截止电压范围内, 其放电比容量为 $179.9 \mathrm{mAh} \cdot \mathrm{g}^{-1}, 80$ 周容量保持率为 $96.52 \%$ 。
\end{abstract}

关键词: 锂离子电池; 高镍正极材料; 无钴化; 循环性能 中图分类号: 0646

\section{1 引言}

目前, 自然能源消耗日益加剧, 由此导致的 环境污染问题也日益严重。因此，清洁二次能 源材料的发展是当前世界范围内备受瞩目的热 点 ${ }^{1-3}$ 。近年来, 绿色二次电池技术的发展突飞猛 进, 其中具有高能量密度的锂离子电池作为储能 装置已广泛应用于现代生活, 在储能电网, 电动 工具, 便捷式移动设备, 以及电动汽车, 电动飞 机, 电动轮船等均有其身影 $4-7$ 。中国对锂离子电 池的发展极为重视并做出了重要规划。其中《中 国制造2025》中对动力电池发展规划的要求是到 2020年, 电池能量密度达到 $300 \mathrm{Wh} \cdot \mathrm{kg}^{-1}$, 到2025年 达到400 Wh $\mathrm{kg}^{-1}$, 到2030年达到 $500 \mathrm{Wh} \cdot \mathrm{kg}^{-1}$; 《汽 车产业中长期发展规划》中则制定目标为到 2020 年, 动力电池单体能量密度达到 $300 \mathrm{Wh} \cdot \mathrm{kg}^{-1}$, 力 争实现 $350 \mathrm{Wh} \cdot \mathrm{kg}^{-1}$, 系统比能量密度力争达到 $260 \mathrm{Wh} \cdot \mathrm{Kg}^{-1}$, 成本降至 1 元 $\cdot \mathrm{Wh}^{-1}$ 以下, 到 2025 年, 动力电池系统比能量达到 $350 \mathrm{Wh} \cdot \mathrm{kg}^{-1}$ 。

想锂离子电池是最具潜力的候选电池体系之 一要实现上述目标, 以高镍层状材料为正极的 ${ }^{8-10}$ 。 高镍层状正极材料具有比能量密度高 (可以达 到 $\left.800 \mathrm{Wh} \cdot \mathrm{kg}^{-1}\right)$, 快充性能良好, 环境友好等 优点 ${ }^{11-13}$ 。而高镍正极材料也存在一些缺点, 比如 在充放电过程中, 由于 $\mathrm{Ni}^{2+}$ 离子半径 $(r=0.069 \mathrm{~nm})$ 和 $\mathrm{Li}^{+}$离子半径 $(r=0.076 \mathrm{~nm})$ 相近, 很容易导致镍 离子向锂位迁移, 造成锂镍混排(即“阳离子混 排”), 从而破坏高镍正极材料结构并诱发电化学
性能衰减 ${ }^{14-16}$ 。

在高镍层状正极材料 $\mathrm{LiNi}_{x} \mathrm{Mn}_{y} \mathrm{Co}_{1-x-y} \mathrm{O}_{2}(x>$ 0.8)中, 不同过渡金属元素起到的作用不同。其 中, $\mathrm{Ni}$ 在电化学充放电过程中存在 $\mathrm{Ni}^{2+} \leftrightarrow \mathrm{Ni}^{3+} \leftrightarrow$ $\mathrm{Ni}^{4+}$ 的氧化还原过程, 这个过程伴随着更多的 $\mathrm{Li}^{+}$ 可逆嵌入脱出晶格, 为高镍正极材料提供了更高 的可逆循环容量 ${ }^{17,18} ; \mathrm{Mn}^{4+}$ 离子在电化学过程中不 发生氧化还原反应, 有助于提高正极材料在电化 学过程中的结构稳定性和热稳定性能 ${ }^{19,20}$; 而 $\mathrm{Co}^{3+}$ 磁矩为 0 , 占据高镍层状结构中过渡金属位置后能 够抑制锂镍混排, 并增强材料导离子能力 ${ }^{21,22}$ 。

钴在高镍正极材料中的作用至关重要, 但是 目前全球范围内钴矿资源紧缺, 存储量仅为 687.5 万吨, 氧化钴价格更是高达 21 万元每吨; 而原材 料成本价格偏高是阻碍高镍正极材料广泛应用的 主要原因之一。因此, 寻找一种资源存储量高、 价格低廉且不影响高镍正极材料的电化学性能的 过渡金属元素替代Co, 是目前商业界以及学术界 共同关注的热点问题。由此, 本研究根据Co 的性 质, 选取 $\mathrm{Cr}^{3+} 、 \mathrm{Cd}^{2+} 、 \mathrm{Zr}^{4+}$ 几种元素替代 $\mathrm{Co}^{3+}$, 进 行无钴化高镍层状材料的相关研究, 分析其可行 性并进行合成工艺优化, 最后对通过合成更高镍 含量的无钴正极材料验证该优化工艺。本实验的 设计思路如图1所示。本文旨在通过共沉淀法合成 高性能的无钴化高镍正极材料, 以期降低锂离子 电池正极材料的成本, 从而促进高镍材料在电动 汽车中的广泛应用。 


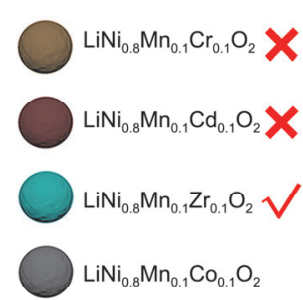

The feasibility analysis of substituting Co with $\mathrm{Cr}$, Cd, or $\mathrm{Zr}$

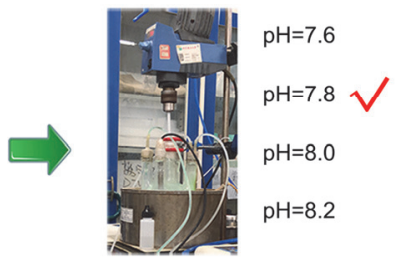

The optimization for Ni-rich precursor Optimized synthetic process for substituting Co with $\mathrm{Zr}$ in Ni-rich cathode

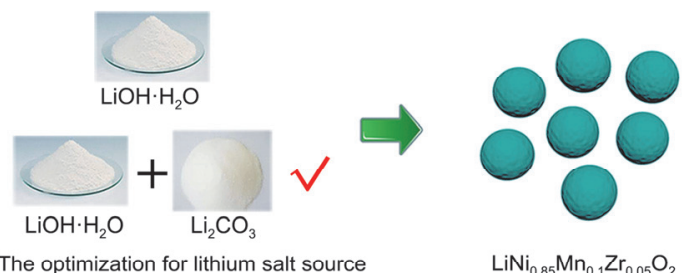

Verifying the feasibility of substituting Co with $\mathrm{Zr}$ in Ni-rich cathode

图 1 实验设计思路

Fig. 1 The design idea of the experiment.

表 1 样品合成条件列表

Table 1 Synthesis conditions for all samples.

\begin{tabular}{|c|c|c|c|c|c|c|}
\hline & Chemical formula & Abbreviation & Precursor & Synthetic method & pH & Lithium salt \\
\hline \multirow{3}{*}{$\begin{array}{l}\text { The feasibility analysis of } \\
\text { substituting Co with } \mathrm{Cr}, \mathrm{Cd}\end{array}$} & $\mathrm{LiNi}_{0.8} \mathrm{Mn}_{0.1} \mathrm{Cr}_{0.1} \mathrm{O}_{2}$ & Cr811 & $\mathrm{Cr}-(\mathrm{OH})_{2}$ & Hydroxide & 11 & $\mathrm{LiOH} \cdot \mathrm{H}_{2} \mathrm{O}$ \\
\hline & $\mathrm{LiNi}_{0.8} \mathrm{Mn}_{0.1} \mathrm{Cd}_{0.1} \mathrm{O}_{2}$ & $\mathrm{Cd} 811$ & $\mathrm{Cd}-(\mathrm{OH})_{2}$ & coprecipitation & & \\
\hline & $\mathrm{LiNi}_{0.8} \mathrm{Mn}_{0.1} \mathrm{Co}_{0.1} \mathrm{O}_{2}$ & Co811 & $\mathrm{Co}-(\mathrm{OH})_{2}$ & & & \\
\hline \multirow{11}{*}{$\begin{array}{l}\text { The feasibility analysis and } \\
\text { synthetic process optimization of } \\
\text { substituting Co with } \mathrm{Zr} \text { in } \\
\text { Ni-rich cathode }\end{array}$} & $\mathrm{LiNi}_{0.8} \mathrm{Mn}_{0.1} \mathrm{Co}_{0.1} \mathrm{O}_{2}$ & $\mathrm{Co}-\mathrm{LiOH}$ & $\mathrm{Co}-\mathrm{CO}_{3}$ & Carbonate & 7.8 & $\mathrm{LiOH} \cdot \mathrm{H}_{2} \mathrm{O}$ \\
\hline & $\mathrm{LiNi}_{0.8} \mathrm{Mn}_{0.1} \mathrm{Zr}_{0.1} \mathrm{O}_{2}$ & Zr-LiOH-7.6 & $\mathrm{Zr}-\mathrm{CO}_{3}$ & coprecipitation & 7.6 & $\mathrm{LiOH} \cdot \mathrm{H}_{2} \mathrm{O}$ \\
\hline & & $\mathrm{Zr}-\mathrm{LiOH}$ & & & 7.8 & \\
\hline & & $\mathrm{Zr}-\mathrm{LiOH}-8.0$ & & & 8.0 & \\
\hline & & $\mathrm{Zr}-\mathrm{LiOH}-8.2$ & & & 8.2 & \\
\hline & $\mathrm{LiNi}_{0.8} \mathrm{Mn}_{0.1} \mathrm{Co}_{0.1} \mathrm{O}_{2}$ & $\mathrm{Co}-\mathrm{LiOH} / \mathrm{Li}_{2} \mathrm{CO}_{3}$ & $\mathrm{Co}-\mathrm{CO}_{3}$ & Carbonate & 7.8 & $\mathrm{LiOH} \cdot \mathrm{H}_{2} \mathrm{O}+$ \\
\hline & & & & coprecipitation & & $\mathrm{Li}_{2} \mathrm{CO}_{3}$ \\
\hline & $\mathrm{LiNi}_{0.8} \mathrm{Mn}_{0.1} \mathrm{Zr}_{0.1} \mathrm{O}_{2}$ & $\mathrm{Zr}-\mathrm{LiOH} / \mathrm{Li}_{2} \mathrm{CO}_{3}-7.6$ & $\mathrm{Zr}-\mathrm{CO}_{3}$ & & 7.6 & $\mathrm{LiOH} \cdot \mathrm{H}_{2} \mathrm{O}+$ \\
\hline & & $\mathrm{Zr}-\mathrm{LiOH} / \mathrm{Li}_{2} \mathrm{CO}_{3}$ & & & 7.8 & $\mathrm{Li}_{2} \mathrm{CO}_{3}$ \\
\hline & & $\mathrm{Zr}-\mathrm{LiOH} / \mathrm{Li}_{2} \mathrm{CO}_{3}-8.0$ & & & 8.0 & \\
\hline & & $\mathrm{Zr}-\mathrm{LiOH} / \mathrm{Li}_{2} \mathrm{CO}_{3}-8.2$ & & & 8.2 & \\
\hline \multirow{4}{*}{$\begin{array}{l}\text { The verification of substituting } \\
\text { Co with } \mathrm{Zr} \text { in Ni-rich cathode }\end{array}$} & $\mathrm{LiNi}_{0.85} \mathrm{Mn}_{0.1} \mathrm{Co}_{0.05} \mathrm{O}_{2}$ & $\mathrm{Co} 8555-\mathrm{LiOH}$ & $\mathrm{Ni}_{0.85} \mathrm{Mn}_{0.1} \mathrm{Co}_{0.05} \mathrm{CO}_{3}$ & Carbonate & 7.8 & $\mathrm{LiOH} \cdot \mathrm{H}_{2} \mathrm{O}$ \\
\hline & $\mathrm{LiNi}_{0.85} \mathrm{Mn}_{0.1} \mathrm{Zr}_{0.05} \mathrm{O}_{2}$ & Zr8555-LiOH & $\mathrm{Ni}_{0.85} \mathrm{Mn}_{0.1} \mathrm{Zr}_{0.05} \mathrm{CO}_{3}$ & coprecipitation & & \\
\hline & $\mathrm{LiNi}_{0.85} \mathrm{Mn}_{0.1} \mathrm{Co}_{0.05} \mathrm{O}_{2}$ & $\mathrm{Co} 8555-\mathrm{LiOH} / \mathrm{Li}_{2} \mathrm{CO}_{3}$ & $\mathrm{Ni}_{0.85} \mathrm{Mn}_{0.1} \mathrm{Co}_{0.05} \mathrm{CO}_{3}$ & & & $\mathrm{LiOH} \cdot \mathrm{H}_{2} \mathrm{O}+$ \\
\hline & $\mathrm{LiNi}_{0.85} \mathrm{Mn}_{0.1} \mathrm{Zr}_{0.05} \mathrm{O}_{2}$ & $\mathrm{Zr} 8555-\mathrm{LiOH} / \mathrm{Li}_{2} \mathrm{CO}_{3}$ & $\mathrm{Ni}_{0.85} \mathrm{Mn}_{0.1} \mathrm{Zr}_{0.05} \mathrm{CO}_{3}$ & & & $\mathrm{Li}_{2} \mathrm{CO}_{3}$ \\
\hline
\end{tabular}

\section{2 实验部分}

\section{1 样品制备}

样品的具体合成条件如表1所列。

通过氢氧化物共沉淀法, 制备 $\mathrm{Ni}_{0.8} \mathrm{Mn}_{0.1} \mathrm{M}^{\prime}{ }_{0.1}(\mathrm{OH})_{2} \quad\left(\mathrm{M}^{\prime}=\mathrm{Cr}, \mathrm{Cd}, \mathrm{Co}\right)$ 前 驱 体。实验步骤如文献所述 ${ }^{16}$ 。通过氢氧化物 共沉淀法得到的前驱体 $\mathrm{Ni}_{0.8} \mathrm{Mn}_{0.1} \mathrm{Cr}_{0.1}(\mathrm{OH})_{2}$, $\mathrm{Ni}_{0.8} \mathrm{Mn}_{0.1} \mathrm{Cd}_{0.1}(\mathrm{OH})_{2}$ 和 $\mathrm{Ni}_{0.8} \mathrm{Mn}_{0.1} \mathrm{Co}_{0.1}(\mathrm{OH})_{2}$ 分别记 为 $\mathrm{Cr}-(\mathrm{OH})_{2}, \mathrm{Cd}-(\mathrm{OH})_{2}$ 和 $\mathrm{Co}-(\mathrm{OH})_{2}$ 。

通过碳酸盐共沉淀方法合成 $\mathrm{Ni}_{0.8} \mathrm{Mn}_{0.1} \mathrm{M}{ }_{0.1} \mathrm{CO}_{3}(\mathrm{M} "=\mathrm{Zr}, \mathrm{Co})$ 前驱体。按照化 学计量比将 $\mathrm{Ni}, \mathrm{Mn}$ 和 $\mathrm{M}$ " $(\mathrm{M}=\mathrm{Co}, \mathrm{Zr})$ 的硫酸盐溶 于去离子水并配置成 $2 \mathrm{~mol} \cdot \mathrm{L}^{-1}$ 溶液 $\mathrm{A}$, 再配置一定 浓度的无水碳酸钠溶液 $\mathrm{B}$, 分别将 $\mathrm{A}$ 和 $\mathrm{B}$ 两种溶液 搅拌 $0.5 \mathrm{~h}$ 后同时泵入 $55^{\circ} \mathrm{C}$ 水浴的圆底烧瓶中, 通 入适量氨水调节 $\mathrm{pH}$ 至 7.8 。进样结束后, 持续摚拌
$2 \mathrm{~h}$ 使前驱体颗粒充分生长, 经去离子水洗涤、过 滤, 将得到的滤渣在真空烘箱中干燥 $12 \mathrm{~h}$, 得到 碳酸盐前驱体, 分别记为 $\mathrm{Zr}-\mathrm{CO}_{3}$ 和 $\mathrm{Co}-\mathrm{CO}_{3}$ 。

本研究中采用两种前驱体混入锂化方法。方 法一采用 $\mathrm{LiOH} \cdot \mathrm{H}_{2} \mathrm{O}$ 作为锂源, 按照 $n_{\mathrm{Li}}: n_{\mathrm{TM}}=$ 1.02:1 (TM为过渡金属元素)的比例将锂盐与前驱 体研磨均匀后, 以 $550{ }^{\circ} \mathrm{C}$ 初烧 $5 \mathrm{~h}$, 之后以 $730{ }^{\circ} \mathrm{C}$ 复烧 $12 \mathrm{~h}$, 得到高镍正极材料。其中, 以氢氧化 物前驱体为原料制得的高镍正极材料记为 $\mathrm{Cr} 811$ 、 Cd811、Co811，以碳酸盐前驱体为原料制得的高 镍正极材料分别记为 $\mathrm{Zr}-\mathrm{LiOH}$ 和 $\mathrm{Co}-\mathrm{LiOH}$ 。方法二 同时使用 $\mathrm{LiOH} \cdot \mathrm{H}_{2} \mathrm{O}$ 和 $\mathrm{Li}_{2} \mathrm{CO}_{3}$ 为锂源, 按照 $n_{\mathrm{Li}}$ : $n_{\mathrm{TM}}=1.02: 1, n_{\mathrm{LiOH} \cdot \mathrm{H}_{2} \mathrm{O}}: n_{\mathrm{Li}_{2} \mathrm{CO}_{3}}=1: 1$ 的比例将锂 盐与碳酸盐前驱体混合研磨均匀, 以 $550{ }^{\circ} \mathrm{C}$ 初烧 $5 \mathrm{~h}$, 之后以 $750{ }^{\circ} \mathrm{C}$ 复烧 $12 \mathrm{~h}$, 得到高镍正极材 料, 分别记为 $\mathrm{Zr}-\mathrm{LiOH} / \mathrm{Li}_{2} \mathrm{CO}_{3}$ 和 $\mathrm{Co}-\mathrm{LiOH} / \mathrm{Li}_{2} \mathrm{CO}_{3}$ 。 
进一步的, 在上述碳酸盐共沉淀方法合成 $\mathrm{Ni}_{0.8} \mathrm{Mn}_{0.1} \mathrm{Zr}_{0.1} \mathrm{CO}_{3}$ 前驱体过程中, 为进一步探 究最佳反应 $\mathrm{pH}$ 值, 分别用氨水调节 $\mathrm{pH}$ 为 7.6 , $7.8,8.0$ 和 8.2 。而后采用方法一和方法二进行锂 化烧结, 其中混入 $\mathrm{LiOH} \cdot \mathrm{H}_{2} \mathrm{O}\left(n_{\mathrm{Li}}: n_{\mathrm{TM}}=1.02: 1\right)$, 经过研磨和高温炦烧 $\left(550{ }^{\circ} \mathrm{C}\right.$ 初烧 $5 \mathrm{~h}, 730{ }^{\circ} \mathrm{C}$ 复 烧 $12 \mathrm{~h}$ ) 得到正极材料, 分别记为 $\mathrm{Zr}-\mathrm{LiOH}-7.6$, $\mathrm{Zr}-\mathrm{LiOH}, \mathrm{Zr}-\mathrm{LiOH}-8.0$ 和Zr-LiOH-8.2; 混入 $\mathrm{LiOH} \cdot \mathrm{H}_{2} \mathrm{O}$ 和 $\mathrm{Li}_{2} \mathrm{CO}_{3}$ 的混合盐 $\left(n_{\mathrm{Li}}: n_{\mathrm{TM}}=1.02: 1\right.$, $\left.n_{\mathrm{LiOH} \cdot \mathrm{H}_{2} \mathrm{O}}: n_{\mathrm{Li}_{2} \mathrm{CO}_{3}}=1: 1\right)$, 经过研磨和高温㷽烧 $\left(550^{\circ} \mathrm{C}\right.$ 初烧 $5 \mathrm{~h}, 750{ }^{\circ} \mathrm{C}$ 复烧 $\left.12 \mathrm{~h}\right)$ 得到的正极材料 分别记为 $\mathrm{Zr}-\mathrm{LiOH} / \mathrm{Li}_{2} \mathrm{CO}_{3}-7.6, \mathrm{Zr}-\mathrm{LiOH} / \mathrm{Li}_{2} \mathrm{CO}_{3}$, $\mathrm{Zr}-\mathrm{LiOH} / \mathrm{Li}_{2} \mathrm{CO}_{3}-8.0$ 和 $\mathrm{Zr}-\mathrm{LiOH} / \mathrm{Li}_{2} \mathrm{CO}_{3}-8.2$ 。

在上述碳酸盐共沉淀方法中, 通过氨水调节 $\mathrm{pH}$ 为7.8, 调整过渡金属离子摩尔比例为 $\mathrm{Ni}: \mathrm{Mn}$ : $\mathrm{M} "=0.85$ : 0.1 : 0.05 , 合成前驱体 $\mathrm{Ni}_{0.85} \mathrm{Mn}_{0.1} \mathrm{Co}_{0.05} \mathrm{CO}_{3}$ 和 $\mathrm{Ni}_{0.85} \mathrm{Mn}_{0.1} \mathrm{Zr}_{0.05} \mathrm{CO}_{3}$ 。混入 锂盐 $\mathrm{LiOH} \cdot \mathrm{H}_{2} \mathrm{O}\left(n_{\mathrm{Li}}: n_{\mathrm{TM}}=1.02: 1\right)$, 经过研磨和 高温煅烧 $\left(550^{\circ} \mathrm{C}\right.$ 初烧 $5 \mathrm{~h}, 730^{\circ} \mathrm{C}$ 复烧 $\left.12 \mathrm{~h}\right)$ 得到的 正极材料记为 Co8555- $\mathrm{LiOH}$, 和Zr8555- $\mathrm{LiOH}$ 。混 入锂 盐 $\mathrm{LiOH} \cdot \mathrm{H}_{2} \mathrm{O}$ 和 $\mathrm{Li}_{2} \mathrm{CO}_{3}$ 的混合盐 $\left(n_{\mathrm{Li}}: n_{\mathrm{TM}}=\right.$ $\left.1.02: 1, n_{\mathrm{LiOH} \cdot \mathrm{H}_{2} \mathrm{O}}: n_{\mathrm{Li}_{2} \mathrm{CO}_{3}}=1: 1\right)$, 经过研磨和煅烧 $\left(550{ }^{\circ} \mathrm{C}\right.$ 初烧 $5 \mathrm{~h}, 750{ }^{\circ} \mathrm{C}$ 复烧 $\left.12 \mathrm{~h}\right)$ 得到的正极材料 记为 $\mathrm{Co} 8555-\mathrm{LiOH} / \mathrm{Li}_{2} \mathrm{CO}_{3}$ 和 $\mathrm{Zr} 8555-\mathrm{LiOH} / \mathrm{Li}_{2} \mathrm{CO}_{3}$ 。

原 料: $\mathrm{MnSO}_{4} \cdot \mathrm{H}_{2} \mathrm{O}$ (分析纯, $99 \%$ ), $\mathrm{CoSO}_{4} \cdot 7 \mathrm{H}_{2} \mathrm{O}$ (分析纯, $99 \%$ ), $\mathrm{NiSO}_{4} \cdot 7 \mathrm{H}_{2} \mathrm{O}$ (分析 纯, $99 \%$ ), $\mathrm{Zr}_{2}\left(\mathrm{SO}_{4}\right)_{2} \cdot 4 \mathrm{H}_{2} \mathrm{O}$ (分析纯, $98 \%$ ), $\mathrm{Cr}_{2}\left(\mathrm{SO}_{4}\right)_{3} \cdot x \mathrm{H}_{2} \mathrm{O}$ (分析纯, $99 \%$ ), $\mathrm{CdSO}_{4} \cdot 8 / 3 \mathrm{H}_{2} \mathrm{O}$ (分析纯, $99 \%$ )。

\section{2 样品表征}

采用旋转阳极X射线衍射仪(XRD, 日本 Rigaku公司, 型号IV-185)分析样品材料结构, 测 试条件: $\mathrm{Cu}$ 靶, $K_{\alpha}$ 射线, 靶电压 $40 \mathrm{kV}$, 靶电流 $40 \mathrm{~mA}$, 扫描范围为 $10^{\circ}-90^{\circ}, 2 \theta$ 扫描速率为 $8\left(^{\circ}\right) \cdot \mathrm{min}^{-1}$ 。采用荷兰公司生产的扫描电镜 (SEM, 型号QUANTA 250), 分析目标产物的颗粒 大小和表面形貌。采用电感偶合等离子体原子发 射光谱(ICP-AES, IRIS Intrepid II), 测量层状氧 化物中各元素的成分比例。

\section{3 电池组装和测试}

将正极活性物质和聚偏氟乙烯(PVDF)以及乙 炔黑按照质量比 $8: 1: 1$ 混合成浆料, 以 $\mathrm{N}$-甲基-2吡咯烷酮(NMP)为粘结剂, 研磨均匀后, 涂布在铝 集流体上, 置于 $80^{\circ} \mathrm{C}$ 烘箱中干燥 $10 \mathrm{~h}$, 经过辊压 裁片, 在手套箱中组装成CR2025型扣式电池。其 中负极为金属锂片, 隔膜为Celgard 2300 多孔复合
聚合物膜, 电解液为 $1 \mathrm{~mol} \cdot \mathrm{L}^{-1}$ 的 $\mathrm{LiPF}_{6}$ 溶解于碳酸 乙烯酯(EC)和碳酸二甲酯 $(\mathrm{DMC})\left(V_{\mathrm{EC}}: V_{\mathrm{DMC}}=1\right.$ : 1)。电池测试在LAND电池测试系统(武汉金诺公 司)上于 $25{ }^{\circ} \mathrm{C}$ 进行恒电流充放电循环测试, 充放 电电压区间为2.75-4.3 V (vs. $\left.\mathrm{Li} / \mathrm{Li}^{+}\right)$, 其中充放电 电流以 $1 C=180 \mathrm{~mA} \cdot \mathrm{g}^{-1}$ 计算。

\section{3 结果与讨论}

\section{1 $\mathrm{Cr}^{3+}$ 和 $\mathrm{Cd}^{2+}$ 掺杂的无钴高镍正极材料}

由于 $\mathrm{Cr}^{3+}$ 的离子半径 $(r=0.0615 \mathrm{~nm})$ 与 $\mathrm{Co}^{3+}$ 离 子半径 $(r=0.0545 \mathrm{~nm})$ 相近, $\mathrm{Cr}$ 与 Co 在元素周期表 中位于同一主族, 且 $\mathrm{Cr}^{3+}$ 掺杂可以有效增强高镍 层状正极材料电化学性能 ${ }^{23}$; 另外, 由于电荷补 偿作用, $\mathrm{Cd}^{2+}$ 掺杂进入层状正极晶格可以提高 $\mathrm{Ni}$ 离子的氧化价态, 从而减少 $\mathrm{Li}^{+} / \mathrm{Ni}^{2+}$ 混排, 因此选 择 $\mathrm{Cr}^{3+}$ 和 $\mathrm{Cd}^{2+}$ 作为替代 $\mathrm{Co}$ 的元素。

$\mathrm{Cr}-(\mathrm{OH})_{2} 、 \mathrm{Cd}-(\mathrm{OH})_{2}$ 和 $\mathrm{Co}-(\mathrm{OH})_{2}$ 三种前驱体材 料的形貌如图 2 中 SEM图所示, $\mathrm{Cr}-(\mathrm{OH})_{2}$ 氢氧化物 前驱体二次颗粒直径 $4 \mu \mathrm{m}$, 且孔洞比较多, 不利 于电化学循环过程中 $\mathrm{Li}^{+}$的可逆嵌脱; $\mathrm{Cd}-(\mathrm{OH})_{2}$ 氢 氧化物前驱体二次颗粒直径为 $8 \mu \mathrm{m}$, 二次颗粒尺 寸均匀但一次颗粒呈现比较大的薄片状结构, 且 一次颗粒的排布无序同样不利 $\mathrm{Li}^{+}$的嵌脱; Co$(\mathrm{OH})_{2}$ 的氢氧化物前驱体二次颗粒直径 $10 \mu \mathrm{m}$, 二 次颗粒尺寸分布均匀且一次颗粒呈放射状向心排 布, 能够致密堆积形成二次颗粒, 有利于充放电 过程中 $\mathrm{Li}^{+}$的迁移。

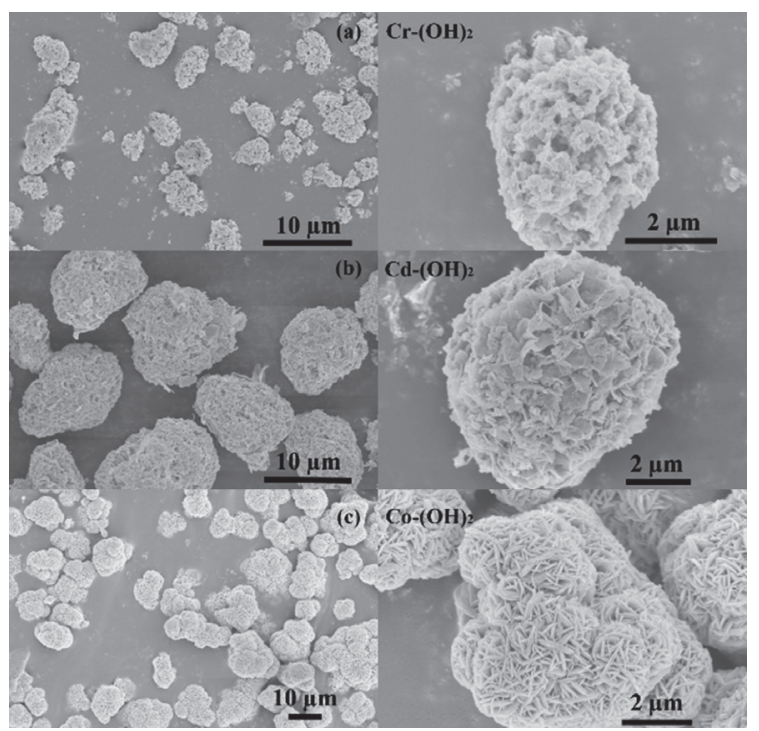

图 2 氢氧化物共沉淀前驱体 $\mathrm{Cr}-(\mathrm{OH})_{2} 、 \mathrm{Cd}-(\mathrm{OH})_{2}$ 、

$\mathrm{Co}-(\mathrm{OH})_{2}$ 的 SEM 图

Fig. 2 SEM images of (a) $\mathrm{Cr}-(\mathrm{OH})_{2}$, (b) $\mathrm{Cd}-(\mathrm{OH})_{2}$, and (c) $\mathrm{Co}-(\mathrm{OH})_{2}$. 
采用电感耦合等离子体原子发射光谱(ICP)定 量分析 $\mathrm{Cr} 811, \mathrm{Cd} 811$ 和 Co811三种正极材料的元素 组成, 其化学式分别为 $\mathrm{LiNi}_{0.792} \mathrm{Mn}_{0.081} \mathrm{Cr}_{0.127} \mathrm{O}_{2}$, $\mathrm{LiNi}_{0.777} \mathrm{Mn}_{0.087} \mathrm{Cd}_{0.136} \mathrm{O}_{2}$ 和 $\mathrm{LiNi}_{0.810} \mathrm{Mn}_{0.093} \mathrm{Co}_{0.097} \mathrm{O}_{2}$, 与 $\mathrm{Ni}: \mathrm{Mn}: \mathrm{M}^{\prime}\left(\mathrm{M}^{\prime}=\mathrm{Cr}, \mathrm{Cd}, \mathrm{Co}\right)=8: 1: 1$ 的情况 基本吻合。在2.75-4.3 V电压范围内测试三种正 极材料的电化学循环性能, 如图3所示, Co811的 初始放电容量 $188.1 \mathrm{mAh} \cdot \mathrm{g}^{-1}$, 循环 100 周容 量保持率为 $87.7 \%$; 而 $\mathrm{Cd} 811$ 的初始放电容量是 $84.3 \mathrm{mAh} \cdot \mathrm{g}^{-1}$, 100周容量保持率 $57.2 \%$; Cr811材 料的初始放电容量只有 $65.9 \mathrm{mAh} \cdot \mathrm{g}^{-1}, 100$ 周容量 保持率仅为 $61 \%$ 。由于 $\mathrm{Cd}$ 和 $\mathrm{Cr}$ 替代 $\mathrm{Co}$ 得到的材料 电化学性能都比较差, 因此, 我们认为 $\mathrm{Cd}$ 和 $\mathrm{Cr}$ 在 高镍三元材料中替代Co是不可行的。

\section{$3.2 \mathrm{Zr}^{4+}$ 掺杂的无钴高镍正极材料}

由于 $\mathrm{Zr}^{4+}$ 离子掺杂高镍正极材料可以有效提 高高镍正极材料结构稳定性, 抑制其阳离子混 排, 提高正极材料导电性, 又因为全球范围内锆 矿 $\left(\mathrm{ZrO}_{2}\right)$ 储量稳定在 7500 万吨, 并且价格低廉, 高 级锆英砂每吨仅 1 万元左右, 远低于钴矿价格 ${ }^{24-26}$ 。 所以 Zr可以作为另一个替代Co的过渡金属元素掺 杂到高镍正极材料中。由于 $\mathrm{Zr}^{4+}$ 沉淀 $\mathrm{pH}$ 值的限 制, 我们采用碳酸盐共沉淀法合成碳酸盐前驱体 $\mathrm{Zr}-\mathrm{CO}_{3}$ 以及其对比样 $\mathrm{Co}-\mathrm{CO}_{3}$ 。通过 SEM测试分析 这两种前驱体材料形貌(图4), $\mathrm{Zr}-\mathrm{CO}_{3}$ 碳酸盐共沉 淀前驱体的颗粒破碎严重, 成球性较差, 相比较 而言 $\mathrm{Co}-\mathrm{CO}_{3}$ 碳酸盐共沉淀前驱体材料二次颗粒成 球度更高, 一次颗粒堆积的更致密。

3.2.1 $\mathrm{Zr}^{4+}$ 掺杂无钻高镍正极材料的锂化工艺优化 ICP测得 $\mathrm{Co}-\mathrm{LiOH}$ 和 Co-LiOH的化学式分别为 $\mathrm{LiNi}_{0.76} \mathrm{Mn}_{0.11} \mathrm{Zr}_{0.13} \mathrm{O}_{2}$, 和 $\mathrm{LiNi}_{0.810} \mathrm{Mn}_{0.094} \mathrm{Co}_{0.096} \mathrm{O}_{2}$, 符合预期设计。对这两种材料进行电化学性能测 试, 如图5和表2所示。在 $0.2 C$ 倍率下, $\mathrm{Co}-\mathrm{LiOH}$

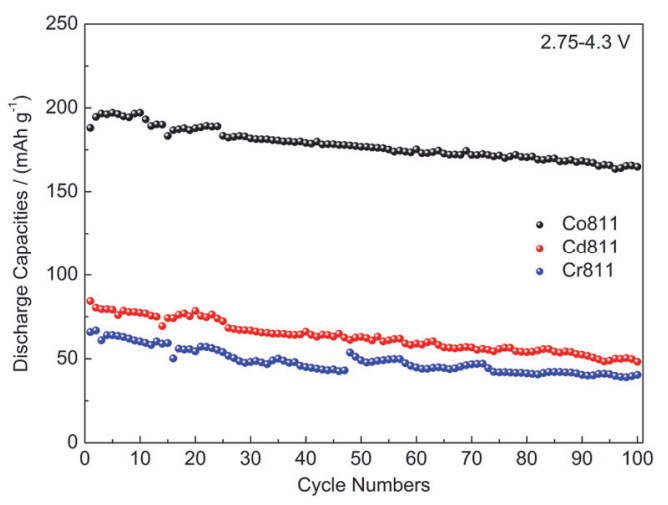

图 $3 \mathrm{Co811}, \mathrm{Cd811}$ 和 $\mathrm{Cr811}$ 的 $0.2 C$ 电化学循环性能图

Fig. 3 Electrochemical cycling performance of Co811, Cd811, and Cr811 at 0.2C.

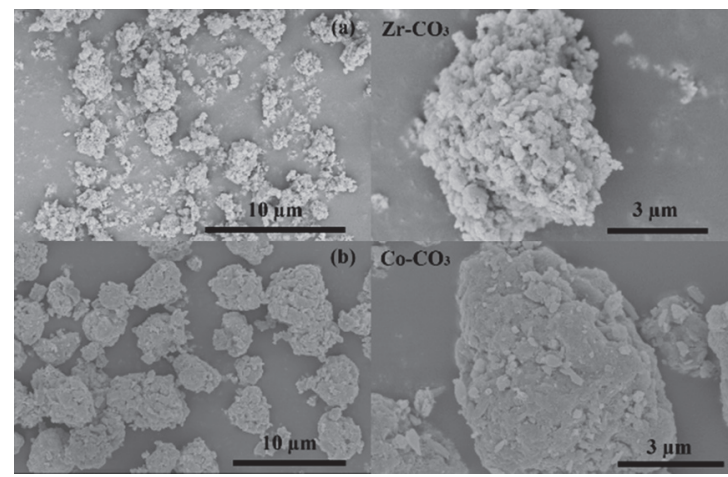

图 4 碳酸盐共沉淀前驱体(a) $\mathrm{Zr}-\mathrm{CO}_{3}$ 和 (b) $\mathrm{Co}-\mathrm{CO}_{3}$ 的 $\mathrm{SEM}$ 图

Fig. 4 SEM images of (a) $\mathrm{Zr}-\mathrm{CO}_{3}$ and (b) $\mathrm{Co}-\mathrm{CO}_{3}$.

在2.75-4.3 V的首周放电比容量为 $157.5 \mathrm{mAh} \cdot \mathrm{g}^{-1}$, 100 周容量保持率 $86.1 \%$ 。碳酸盐共沉淀法合成的 Co-LiOH的电化学性能略差于氢氧化物共沉淀法得 到的Co 811 , 因此本实验后面进一步探究了碳酸盐 共沉淀合成高镍材料前驱体的优化条件。Zr-LiOH 正极材料的首周放电容量为 $145.8 \mathrm{mAh} \cdot \mathrm{g}^{-1}, 100$ 周 容量保持率为 $87.04 \%$, 虽然其放电比容量略低于 $\mathrm{Co}-\mathrm{LiOH}$, 但是其容量保持率却有所改善。

对 Co-LiOH和Zr-LiOH进行倍率性能测试(在 $0.2 C 、 0.5 C 、 1 C 、 2 C 、 5 C$ 和 $10 C$ 分别循环 3 周, 然后 恢复至 $0.2 C$ 循环 3 周, 再在 $1 C$ 循环 80 周), 结果如图 $5 \mathrm{~b}$ 所示 。 $\mathrm{Co}-\mathrm{LiOH}$ 在 $0.2 C 、 0.5 C 、 1 C 、 2 C 、 5 C$ 和 $10 C$ 的放电容量分别为 $160 、 156.2 、 149.9$ 、 144.7、133.5、121.6 $\mathrm{mAh} \cdot \mathrm{g}^{-1}$, 并在恢复 $0.2 C$ 循环 时表现出 $160.9 \mathrm{mAh} \cdot \mathrm{g}^{-1}$ 的放电容量。之后的 80 周 $1 C$ 循环中, $\mathrm{Co}-\mathrm{LiOH}$ 的初始放电容量和 80 周容量 保持率分别为 $147.8 \mathrm{mAh} \mathrm{g}^{-1}$ 和 $91.14 \%$ 。相比之 下, $\mathrm{Zr}-\mathrm{LiOH}$ 的倍率性能略显逊色, 在倍率充放电 $0.2 C 、 0.5 C 、 1 C 、 2 C 、 5 C$ 和 $10 C$ 分 别 为 146.5 、 $132.8 、 118.2 、 104.9 、 73.9 、 48.6 \mathrm{mAh} \cdot \mathrm{g}^{-1}$, 并在恢 复 $0.2 C$ 循环时表现出 $141 \mathrm{mAh} \cdot \mathrm{g}^{-1}$ 的放电容量。在 接下来的 $1 C$ 循环中, $\mathrm{Zr}-\mathrm{LiOH}$ 初始放电容量和 80 周容量保持率分别为 $126.8 \mathrm{mAh} \cdot \mathrm{g}^{-1}$ 和 $90.46 \%$ 。可 以看到, $\mathrm{Zr}$ 替代 $\mathrm{Co}$ 合成的 811 型高镍材料, 虽然放 电容量仍然不足以与NCM811材料謧美, 但其循 环稳定性比较好, 因此仍有进一步探究Zr替代Co 掺杂进入高镍正极材料可行性的必要性。

不同锂源的选择会影响前驱体材料转化成层 状正极材料的结晶过程, 从而影响成品材料的电 化学性能。以 $\mathrm{LiOH} \cdot \mathrm{H}_{2} \mathrm{O}$ 为锂盐时, 前驱体材料锂 化结晶转变成层状正极材料所需要的温度相比于 以 $\mathrm{Li}_{2} \mathrm{CO}_{3}$ 为锂盐的情况所需的温度低, 因而一般 以 $\mathrm{LiOH} \cdot \mathrm{H}_{2} \mathrm{O}$ 为锂源合成三元正极材料所需的煅烧 

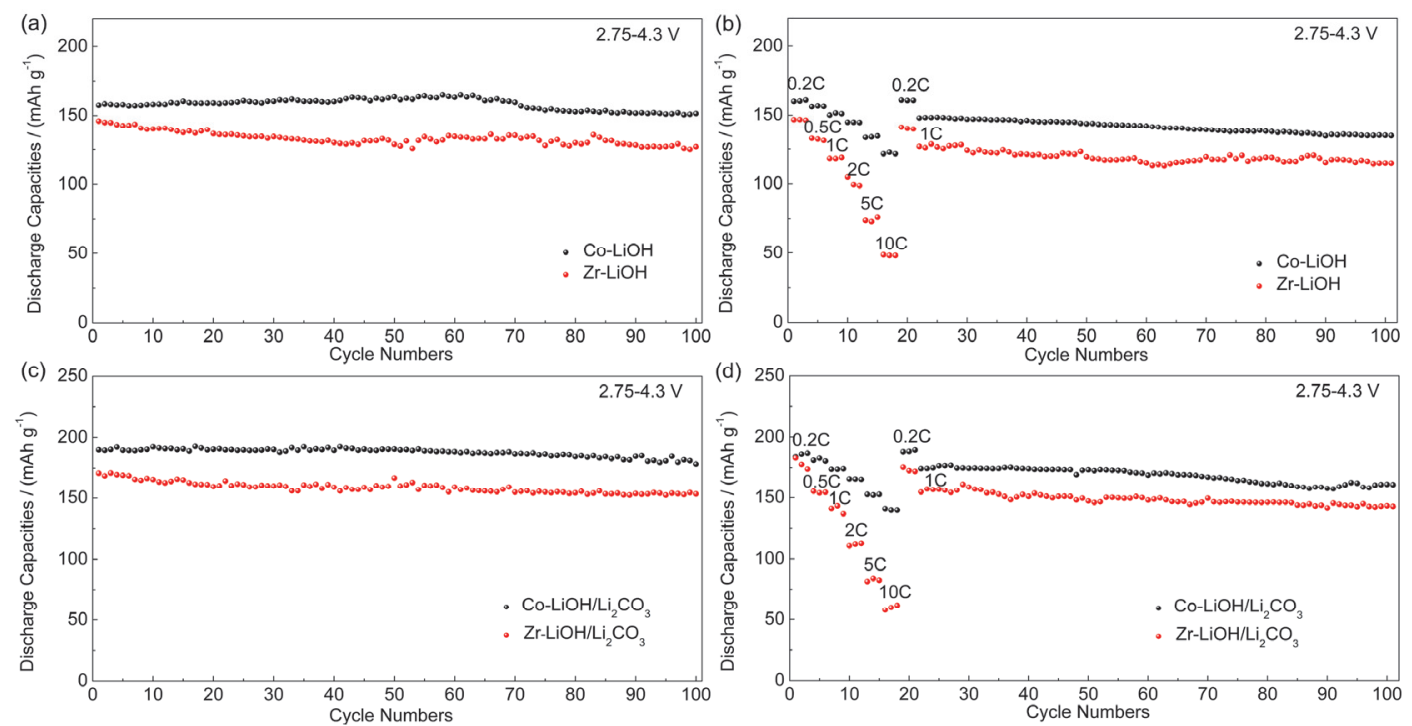

图 $5 \mathrm{Co}-\mathrm{LiOH}$ 和 $\mathrm{Zr}-\mathrm{LiOH}$ 的(a) $0.2 \mathrm{C}$ 长循环测试图和(b) 倍率性能测试图; $\mathrm{Co}-\mathrm{LiOH} / \mathrm{Li}_{2} \mathrm{CO}_{3}$ 和 $\mathrm{Zr}-\mathrm{LiOH} / \mathrm{Li}_{2} \mathrm{CO}_{3}$ 的 (c) $0.2 \mathrm{C}$ 长循环测试图和(d)倍率性能测试图

Fig. 5 (a) Electrochemical cycling performance at $0.2 \mathrm{C}$ and (b) Rate performance of $\mathrm{Co}-\mathrm{LiOH}$ and $\mathrm{Zr}-\mathrm{LiOH}$; Electrochemical cycling performance at $0.2 \mathrm{C}$ and (b) Rate performance of $\mathrm{Co}-\mathrm{LiOH} / \mathrm{Li}_{2} \mathrm{CO}_{3}$ and $\mathrm{Zr}-\mathrm{LiOH} / \mathrm{Li}_{2} \mathrm{CO}_{3}$.

表 $2 \mathrm{Co}-\mathrm{LiOH}, \mathrm{Zr}-\mathrm{LiOH}, \mathrm{Co}-\mathrm{LiOH} / \mathrm{Li}_{2} \mathrm{CO}_{3}$ 和 $\mathrm{Zr}-\mathrm{LiOH} / \mathrm{Li}_{2} \mathrm{CO}_{3}$ 的电化学性能表

Table 2 Electrochemical performances for $\mathrm{Co}-\mathrm{LiOH}, \mathrm{Zr}-\mathrm{LiOH}, \mathrm{Co}-\mathrm{LiOH} / \mathrm{Li}_{2} \mathrm{CO}_{3}$ and $\mathrm{Zr}-\mathrm{LiOH} / \mathrm{Li}_{2} \mathrm{CO}_{3}$.

\begin{tabular}{|c|c|c|c|c|c|c|c|}
\hline Samples & $\begin{array}{l}\text { The initial discharge } \\
\text { capacity at } 0.2 C \\
\text { (coulombic efficiency) }\end{array}$ & $\begin{array}{c}\text { The highest } \\
\text { discharge capacity } \\
\left(\mathrm{mAh} \cdot \mathrm{g}^{-1}\right)\end{array}$ & $\begin{array}{c}\text { 100th discharge } \\
\text { capacity at } 0.2 C \\
\left(\mathrm{mAh} \cdot \mathrm{g}^{-1}\right)\end{array}$ & $\begin{array}{l}100 \text { th capacity } \\
\text { retention at } 0.2 C\end{array}$ & $\begin{array}{c}\text { Discharge } \\
\text { capacity at } 10 C \\
\left(\mathrm{mAh} \cdot \mathrm{g}^{-1}\right) \\
\end{array}$ & $\begin{array}{c}\text { The initial discharge } \\
\text { capacity at } 1 C \\
\left(\mathrm{mAh} \cdot \mathrm{g}^{-1}\right)\end{array}$ & $\begin{array}{l}80 \text { th capacity } \\
\text { retention at } 1 C\end{array}$ \\
\hline $\mathrm{Co}-\mathrm{LiOH}$ & $157.5(79.9 \%)$ & 165 & 151.4 & $86.1 \%$ & 121.6 & 147.8 & $91.14 \%$ \\
\hline $\mathrm{Zr}-\mathrm{LiOH}$ & $145.8(78.7 \%)$ & 145.8 & 126.9 & $87.04 \%$ & 48.6 & 126.8 & $90.46 \%$ \\
\hline $\mathrm{Co}-\mathrm{LiOH} / \mathrm{Li}_{2} \mathrm{CO}_{3}$ & $190(82.7 \%)$ & 192.8 & 178.3 & $93.84 \%$ & 140.8 & 174.2 & $92.31 \%$ \\
\hline $\mathrm{Zr}-\mathrm{LiOH} / \mathrm{Li}_{2} \mathrm{CO}_{3}$ & $170.8(80.1 \%)$ & 170.8 & 153.4 & $89.81 \%$ & 57.8 & 154.6 & $92.24 \%$ \\
\hline
\end{tabular}

温度低于以 $\mathrm{L}_{\mathrm{i} 2} \mathrm{CO}_{3}$ 为锂源所需的煅烧温度。在加 热过程中 $\mathrm{LiOH} \cdot \mathrm{H}_{2} \mathrm{O}$ 会发生分解形成 $\mathrm{Li}_{2} \mathrm{O}$, 导致以 $\mathrm{LiOH} \cdot \mathrm{H}_{2} \mathrm{O}$ 为锂盐形成的高镍正极材料二次颗粒表 面残锂相多于以 $\mathrm{Li}_{2} \mathrm{CO}_{3}$ 为锂盐合成的高镍正极材 料二次颗粒的表面残锂相 ${ }^{27}$ 。为了进一步优化无 钴高镍正极材料的性能, 我们选择不同锂盐为锂 源合成高镍正极材料, 并分析其电化学性能。

$\mathrm{Zr}-\mathrm{LiOH} / \mathrm{Li}_{2} \mathrm{CO}_{3}$ 和 $\mathrm{Co}-\mathrm{LiOH} / \mathrm{Li}_{2} \mathrm{CO}_{3}$ 在 $0.2 \mathrm{C}$, 2.75-4.3 V电压范围内的电化学性能如图 $5 \mathrm{c}$ 和表2 所示。 $\mathrm{Co}-\mathrm{LiOH} / \mathrm{Li}_{2} \mathrm{CO}_{3}$ 在 2.75-4.3 V的初始放电容 量 $190 \mathrm{mAh} \cdot \mathrm{g}^{-1}$, 100周容量保持率 $93.84 \%$ 。而Zr$\mathrm{LiOH} / \mathrm{Li}_{2} \mathrm{CO}_{3}$ 正极材料的首周放电容量 170.8 $\mathrm{mAh} \cdot \mathrm{g}^{-1}, 100$ 周容量保持率为 $89.81 \%$ 。两种材料 在不同倍率下的充放电性能如图 $5 \mathrm{~d}$ 所示, 在电压 $2.75-4.3 \mathrm{~V}$ 范围内, $\mathrm{Co}-\mathrm{LiOH} / \mathrm{Li}_{2} \mathrm{CO}_{3}$ 在 $0.2 C 、 0.5 C$ 、 $1 C 、 2 C 、 5 C$ 和 $10 C$ 倍率下的放电容量分别为 184 、 181.2、173.7、165.7、152.6和 $140.8 \mathrm{mAh} \cdot \mathrm{g}^{-1}$, 并 在恢复 $0.2 C$ 循环时表现出 $188 \mathrm{mAh} \cdot \mathrm{g}^{-1}$ 的放电容
量。相比之下, $\mathrm{Zr}-\mathrm{LiOH} / \mathrm{Li}_{2} \mathrm{CO}_{3}$ 的倍率性能略 差, 在倍率 $0.2 C 、 0.5 C 、 1 C 、 2 C 、 5 C$ 和 $10 C$ 下的 放电容量分别为 $183 、 155.2 、 141 、 110.7 、 81.4$ 和 $57.8 \mathrm{mAh} \cdot \mathrm{g}^{-1}$, 并在恢复 $0.2 C$ 循环时表现出 175.5 $\mathrm{mAh} \cdot \mathrm{g}^{-1}$ 的放电容量。

在接下来的 $1 C$ 循环中, $\mathrm{Co}-\mathrm{LiOH} / \mathrm{Li}_{2} \mathrm{CO}_{3}$ 和 $\mathrm{Zr}-$ $\mathrm{LiOH} / \mathrm{Li}_{2} \mathrm{CO}_{3}$ 都展现了较好的容量保持率, Co$\mathrm{LiOH} / \mathrm{Li}_{2} \mathrm{CO}_{3}$ 的初始 $1 C$ 放电容量和 80 周容量保持 率分别为 $174.2 \mathrm{mAh} \cdot \mathrm{g}^{-1}$ 和 $92.31 \% ; \mathrm{Zr}-\mathrm{LiOH} /$ $\mathrm{Li}_{2} \mathrm{CO}_{3}$ 的初始 $1 C$ 放电容量和 80 周容量保持率分别 $154.6 \mathrm{mAh} \cdot \mathrm{g}^{-1}$ 和 $92.24 \%$ 。可以看 到 $\mathrm{Zr}-\mathrm{LiOH} /$ $\mathrm{Li}_{2} \mathrm{CO}_{3}$ 的电化学循环稳定性能优于Co$\mathrm{LiOH} / \mathrm{Li}_{2} \mathrm{CO}_{3}$ 。由于以 $\mathrm{LiOH} \cdot \mathrm{H}_{2} \mathrm{O}$ 和 $\mathrm{Li}_{2} \mathrm{CO}_{3}$ 混合盐 作为锂源得到高镍正极材料的锂化结晶程度 好, 二次颗粒表面残锂相相对较少, 所以 Zr$\mathrm{LiOH} / \mathrm{Li}_{2} \mathrm{CO}_{3}$ 的电化学性能优于 $\mathrm{Zr}-\mathrm{LiOH}$ 。

3.2.2 $\mathrm{Zr}^{4+}$ 掺杂无钴高镍正极合成过程 $\mathrm{pH}$ 优化 在碳酸盐共沉淀合成前驱体过程中, $\mathrm{pH}$ 值对 
材料形貌以及性能影响很大, $\mathrm{pH}$ 值过低会导致在 共沉淀体系中过渡金属离子络合平衡占据主导, 晶体生长速度快, 成核相对缓慢, 从而导致颗粒 形貌过大; 而若 $\mathrm{pH}$ 值过高, 则会使得体系中碳酸 盐沉淀过饱和度增大, 成核速度快, 晶核来不及 长大, 从而得到的材料颗粒尺寸较小 28-30。因此 应进一步探究 $Z r$ 掺杂的无钴高镍正极材料合成的 最优 $\mathrm{pH}$ 条件。通过调节氨水用量控制碳酸盐共沉 淀合成过程中的 $\mathrm{pH}$ 分别为 $7.6 、 7.8 、 8.0$ 和 8.2 , 并 探究此种情况下合成的材料在 2.75-4.3 V, 0.2C测 试条件下测得的电化学性能, 如图6所示。

如图6a和表3所示, $\mathrm{Zr}-\mathrm{LiOH}-7.6$ 的初始放电 比容量为 $151.6 \mathrm{mAh} \cdot \mathrm{g}^{-1}$, 经过活化后最高放电比 容量为 $154.3 \mathrm{mAh} \cdot \mathrm{g}^{-1}$ (第5周), 循环70周后容量保 持率为 $74.4 \%$; $\mathrm{Zr}-\mathrm{LiOH}$ 的初始放电容量 145.8 $\mathrm{mAh} \cdot \mathrm{g}^{-1}$ (首周为最高放电容量), 循环70周容量保 持率为 $92.73 \%$; Zr-LiOH-8.0的初始放电容量

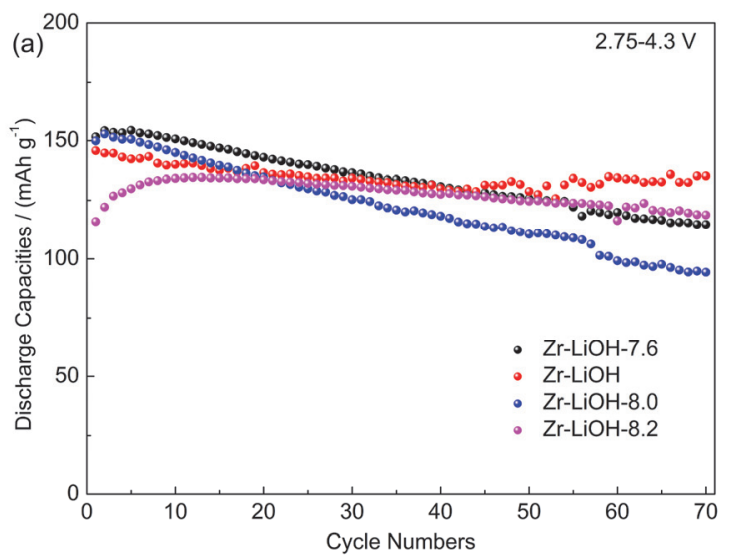

$149.9 \mathrm{mAh} \cdot \mathrm{g}^{-1}$, 最高放电容量为 $152.8 \mathrm{mAh} \cdot \mathrm{g}^{-1}$ (第 2 周), 循环70周容量保持率为 $61.71 \%$; $\mathrm{Zr}-\mathrm{LiOH}-$ 8.2 的初始放电容量 $116 \mathrm{mAh} \mathrm{g}^{-1}$, 最高放电容量为 $134.6 \mathrm{mAh} \cdot \mathrm{g}^{-1}$ (第12周), 循环70周容量保持率为 $88.26 \%$ 。由此可知, Zr-LiOH-7.6的初始放电容量 最高, 但循环稳定性较差; $\mathrm{Zr}-\mathrm{LiOH}-8.0$ 的放电容 量和循环性能均较为恶劣; 虽然Zr-LiOH-8.2的容 量保持率高, 但是其放电容量整体偏低; 而ZrLiOH的放电容量和循环容量保持率都比较理想。

使用 $\mathrm{LiOH} \cdot \mathrm{H}_{2} \mathrm{O}$ 和 $\mathrm{Li}_{2} \mathrm{CO}_{3}$ 混合锂源锂化得到的 高镍正极材料的电化学性能如图 $6 \mathrm{~b}$ 和表3所示。 $\mathrm{Zr}-\mathrm{LiOH} / \mathrm{Li}_{2} \mathrm{CO}_{3}-7.6$ 的 初始放电容量 157.7 $\mathrm{mAh} \cdot \mathrm{g}^{-1}$, 最高放电容量为 $160.3 \mathrm{mAh} \cdot \mathrm{g}^{-1}$ (第5 周), 循环 70 周容量保持率为 $86.59 \%$; $\mathrm{Zr}$ $\mathrm{LiOH} / \mathrm{Li}_{2} \mathrm{CO}_{3}$ 的初始放电容量 $170.8 \mathrm{mAh} \cdot \mathrm{g}^{-1}$, 最高 放电容量为 $171 \mathrm{mAh} \cdot \mathrm{g}^{-1}$ (第3周), 循环70周容量 保持率为 $93.16 \% ; \mathrm{Zr}-\mathrm{LiOH} / \mathrm{Li}_{2} \mathrm{CO}_{3}-8.0$ 的初始放

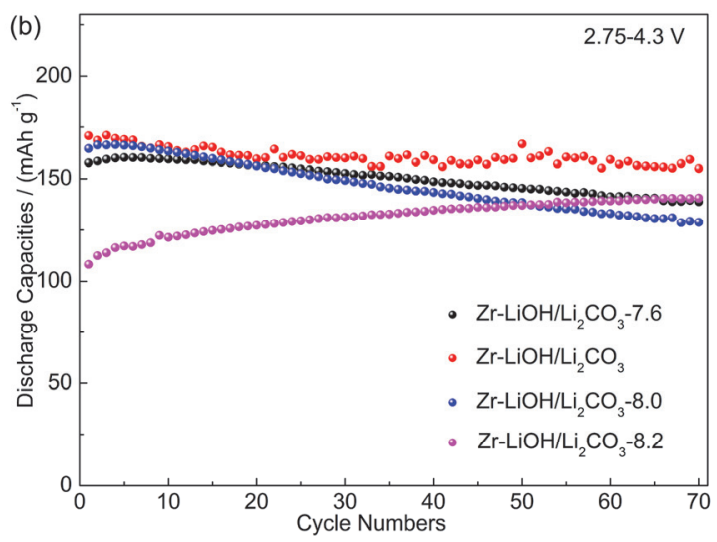

图 6 (a) $\mathrm{Zr}-\mathrm{LiOH}-7.6, \mathrm{Zr}-\mathrm{LiOH}, \mathrm{Zr}-\mathrm{LiOH}-8.0$ 和 $\mathrm{Zr}-\mathrm{LiOH}-8.2 ;(b) \mathrm{Zr}-\mathrm{LiOH} / \mathrm{Li}_{2} \mathrm{CO}_{3}-7.6, \mathrm{Zr}-\mathrm{LiOH} / \mathrm{Li}_{2} \mathrm{CO}_{3}$, $\mathrm{Zr}-\mathrm{LiOH} / \mathrm{Li}_{2} \mathrm{CO}_{3}-8.0$ 和 $\mathrm{Zr}-\mathrm{LiOH} / \mathrm{Li}_{2} \mathrm{CO}_{3}-8.2$ 在 $0.2 \mathrm{C}$ 的电化学循环性能图

Fig. 6 Electrochemical cycling performance of (a) $\mathrm{Zr}-\mathrm{LiOH}-7.6, \mathrm{Zr}-\mathrm{LiOH}, \mathrm{Zr}-\mathrm{LiOH}-8.0$, and $\mathrm{Zr}-\mathrm{LiOH}-8.2$;

(b) $\mathrm{Zr}-\mathrm{LiOH} / \mathrm{Li}_{2} \mathrm{CO}_{3}-7.6, \mathrm{Zr}-\mathrm{LiOH} / \mathrm{Li}_{2} \mathrm{CO}_{3}, \mathrm{Zr}-\mathrm{LiOH}_{/} \mathrm{Li}_{2} \mathrm{CO}_{3}-8.0$, and $\mathrm{Zr}-\mathrm{LiOH} / \mathrm{Li}_{2} \mathrm{CO}_{3}-8.2$ at $0.2 C$.

表 3 在不同 $\mathbf{p H}$ 值条件下合成的 $\mathrm{Zr}^{4+}$ 掺杂的无钴高镍正极材料的电化学性能

Table 3 Electrochemical performances of $\mathrm{Zr}^{4+}$ doped Cobalt-free Ni-rich cathode materials synthesized at different $\mathrm{pH}$.

\begin{tabular}{ccccc}
\hline Samples & $\begin{array}{c}\text { The initial discharge capacity } \\
\left(\mathrm{mAh} \cdot \mathrm{g}^{-1}\right)(\text { coulombic efficiency) }\end{array}$ & $\begin{array}{c}\text { The highest discharge capacity } \\
\left(\mathrm{mAh} \cdot \mathrm{g}^{-1}\right)\end{array}$ & $\begin{array}{c}70^{\text {th }} \text { discharge capacity } \\
\left(\mathrm{mAh} \cdot \mathrm{g}^{-1}\right)\end{array}$ & 70th capacity retention \\
\hline $\mathrm{Zr}-\mathrm{LiOH}-7.6$ & $151.6(74.1 \%)$ & 154.2 & 114.8 & $74.45 \%$ \\
$\mathrm{Zr}-\mathrm{LiOH}$ & $145.8(78.7 \%)$ & 145.8 & 135.2 & $92.73 \%$ \\
$\mathrm{Zr}-\mathrm{LiOH}-8.0$ & $149.9(75.3 \%)$ & 152.8 & 94.3 & $61.71 \%$ \\
$\mathrm{Zr}-\mathrm{LiOH}-8.2$ & $116(67.6 \%)$ & 134.6 & 118.8 & $88.26 \%$ \\
$\mathrm{Zr}-\mathrm{LiOH} / \mathrm{Li}{ }_{2} \mathrm{CO}_{3}-7.6$ & $157.7(68.7 \%)$ & 160.3 & 138.8 & $86.59 \%$ \\
$\mathrm{Zr}-\mathrm{LiOH} / \mathrm{Li}_{2} \mathrm{CO}_{3}$ & $170.8(80.1 \%)$ & 171 & 154.9 & $90.58 \%$ \\
$\mathrm{Zr}-\mathrm{LiOH} / \mathrm{Li}_{2} \mathrm{CO}_{3}-8.0$ & $164.6(77.1 \%)$ & 166.5 & 128.8 & $77.36 \%$ \\
$\mathrm{Zr}-\mathrm{LiOH} / \mathrm{Li}_{2} \mathrm{CO}_{3}-8.2$ & $108.1(51.3 \%)$ & 140.6 & 140.6 & $100 \%$ \\
\hline
\end{tabular}


电容量164.6 $\mathrm{mAh} \cdot \mathrm{g}^{-1}$, 最高放电容量为 166.5 $\mathrm{mAh} \cdot \mathrm{g}^{-1}$ (第4周), 循环70周容量保持率为 $77.36 \%$; $\mathrm{Zr}-\mathrm{LiOH} / \mathrm{Li}_{2} \mathrm{CO}_{3}-8.2$ 的初始放电容量 $108.1 \mathrm{mAh} \cdot \mathrm{g}^{-1}$, 最高放电容量为 $140.6 \mathrm{mAh} \cdot \mathrm{g}^{-1}$ (第70周)。由此可知, $\mathrm{Zr}-\mathrm{LiOH} / \mathrm{Li}_{2} \mathrm{CO}_{3}$ 正极材料 的放电容量和循环稳定性能都是最佳的。相对比 与 $\mathrm{LiNi}_{0.8} \mathrm{Co}_{0.1} \mathrm{Mn}_{0.1} \mathrm{O}_{2}$ 正极材料, $\mathrm{Zr}$ 掺杂形成的 无钴正极材料放电容量偏低, 但电化学循环性能 良好。

通过电化学性能的测试分析, 可以认定 7.8 是 碳酸盐共沉淀合成 $Z r$ 掺杂高镍正极前驱体材料的 最适宜 $\mathrm{pH}$ 值, 此时碳酸盐沉淀的成核和晶体生长 速度达到平衡, 得到的材料中各过渡金属元素可 以均匀沉淀。因而在采用不同锂源的高镍正极材 料中, 在 $\mathrm{pH}=7.8$ 合成的 $\mathrm{Zr}-\mathrm{LiOH}$ 和 $\mathrm{Zr}-\mathrm{LiOH} /$ $\mathrm{Li}_{2} \mathrm{CO}_{3}$ 电化学性能都是最优秀的。另外, 通过比 较不同 $\mathrm{pH}$ 值合成的前驱体材料分别以 $\mathrm{LiOH} \cdot \mathrm{H}_{2} \mathrm{O}$ 和 $\mathrm{LiOH} \cdot \mathrm{H}_{2} \mathrm{O}$ 和 $\mathrm{Li}_{2} \mathrm{CO}_{3}$ 混合盐作为锂源, 经过高温 煅烧得到的正极材料的电化学性能发现, 所有以 混合盐作为锂源得到的高镍正极材料的电化学性 能都优于单独使用 $\mathrm{LiOH} \cdot \mathrm{H}_{2} \mathrm{O}$ 作为锂盐的正极材 料。这是因为以混合锂盐作为锂盐的无钴高镍正 极材料的锂化结晶程度最好, 表面残锂相最少, 所以经过合成工艺优化得到的 $\mathrm{Zr}-\mathrm{LiOH} / \mathrm{Li}_{2} \mathrm{CO}_{3}$ 的 电化学性能最优。

\subsection{3 合成更高镍含量的无钴正极材料验证}

通过合成更高镍含量的无钴高镍正极材料 $\mathrm{LiNi}_{0.85} \mathrm{Mn}_{0.1} \mathrm{Zr}_{0.05} \mathrm{O}_{2}$ 来验证优化合成工艺的可行 性。镍含量更高的正极材料具有更高的放电比容 量, 但是高镍正极材料的结构稳定性却随着镍含 量的增加而降低。首先通过XRD分析 $\mathrm{Co}-\mathrm{LiOH}$, $\mathrm{Zr} 8555-\mathrm{LiOH}, \mathrm{Co} 8555-\mathrm{LiOH} / \mathrm{Li}_{2} \mathrm{CO}_{3}$ 和Zr8555$\mathrm{LiOH} / \mathrm{Li}_{2} \mathrm{CO}_{3}$ 四种材料的晶体结构。如图7所 示, 这四种材料的XRD主峰位置与图7下方坚线 对应的层状结构 $\mathrm{LiNiO}_{2}$ (PDF\#09-063)吻合, 说明 通过碳酸盐共沉淀 $(\mathrm{pH}=7.8)$ 合成前驱体再经过锂 化耘烧可以得到空间群为 $R \overline{3} m$ 的层状正极材 料, 且四种材料的(108)和(110)峰, 以及(006)和 (102)峰之间的分峰清晰明显, 表明其层状晶格结 构良好 ${ }^{31}$ 。四种材料的XRD图谱都没有杂峰存 在, 说明合成过程中过渡金属离子均掺杂进入层 状结构内部, 而没有形成其他杂相。

进一步通过SEM和元素面扫分析Zr8555$\mathrm{LiOH} / \mathrm{Li}_{2} \mathrm{CO}_{3}$ 的形貌以及过渡金属元素分布情 况, 如图8所示。可以看到 $\mathrm{Zr} 8555-\mathrm{LiOH} / \mathrm{Li}_{2} \mathrm{CO}_{3}$ 正 极材料二次颗粒形貌为直径 $10 \mu \mathrm{m}$ 的球形颗粒。其

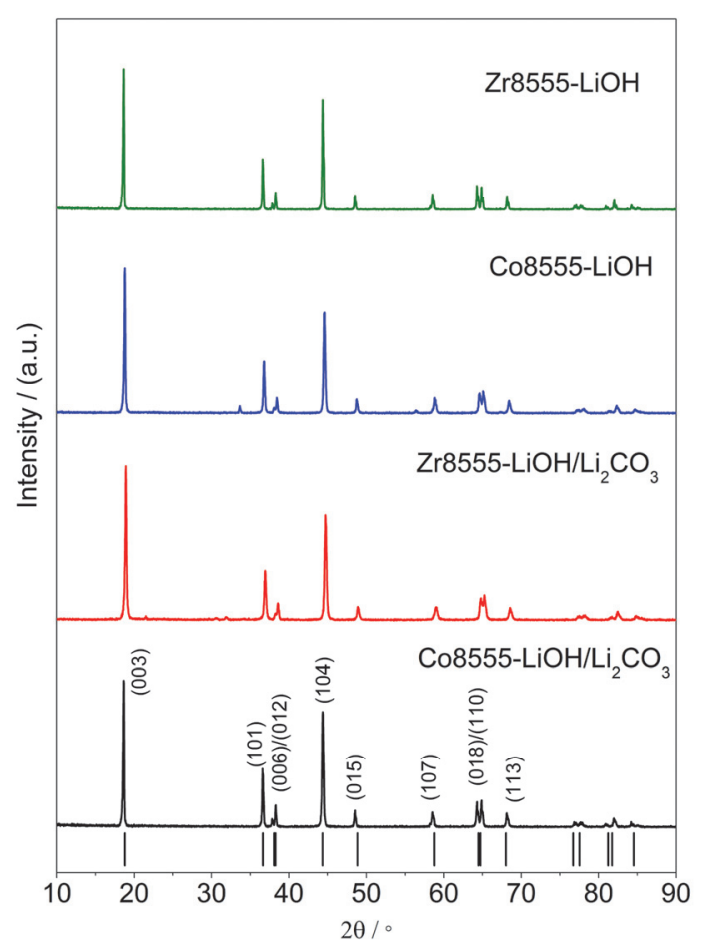

图 7 Co8555-LiOH, Zr8555-LiOH, Co8555-LiOH/

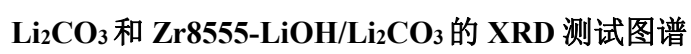

Fig. 7 The XRD patterns of Co8555-LiOH, Zr8555-LiOH, $\mathrm{Co8555}-\mathrm{LiOH} / \mathrm{Li}_{2} \mathrm{CO}_{3}$ and $\mathrm{Zr855}-\mathrm{LiOH} / \mathrm{Li}_{2} \mathrm{CO}_{3}$.

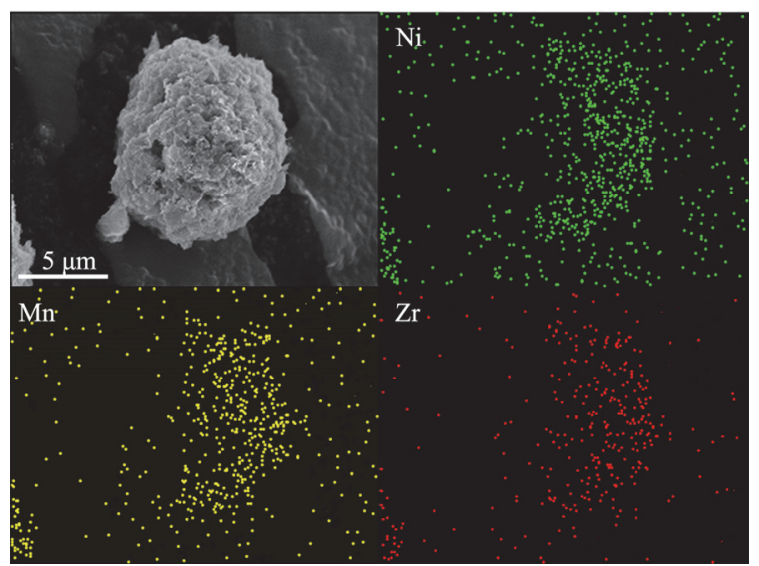

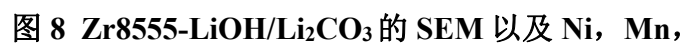
$\mathrm{Zr}$ 的面扫 $\mathrm{EDS}$ 图

Fig. 8 The SEM image and Ni, Mn, $\mathrm{Zr}$ elemental

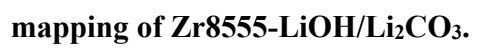

过渡金属元素 $\mathrm{Ni} 、 \mathrm{Mn}$ 和 $\mathrm{Zr}$ 均匀的分布在二次颗粒 表面, 这也表明了 $\mathrm{Ni}, \mathrm{Mn}$ 和 $\mathrm{Zr}$ 三种过渡金属元素 在碳酸盐共沉淀过程中可以均匀的沉淀在高镍正 极前驱体材料中, 再经由锂化㷽烧均匀的分布在 高镍正极材料二次颗粒中。

分别在2.75-4.3 V电压范围内, 以 $0.2 C$ 测试不 同锂化方式得到的Co8555-LiOH、Zr8555-LiOH、 $\mathrm{Co} 8555-\mathrm{LiOH} / \mathrm{Li}_{2} \mathrm{CO}_{3}$ 和 $\mathrm{Zr} 8555-\mathrm{LiOH} / \mathrm{Li}_{2} \mathrm{CO}_{3}$ 四 种 

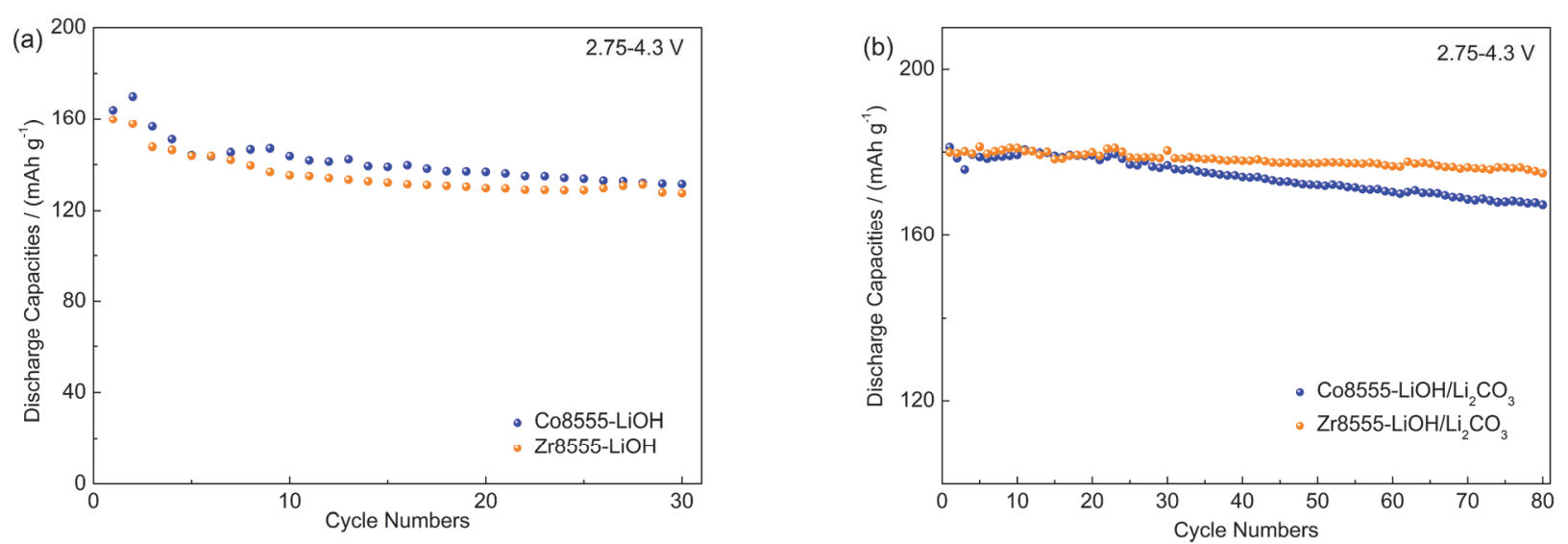

图 9 (a) Co8555- LiOH 和 $\mathrm{Zr8555}-\mathrm{LiOH;}$; (b) $\mathrm{Co8555}-\mathrm{LiOH} / \mathrm{Li}_{2} \mathrm{CO}_{3}$ 和 $\mathrm{Zr8555}-\mathrm{LiOH} / \mathrm{Li}_{2} \mathrm{CO}_{3}$ 在 $0.2 \mathrm{C}$ 的电化学循环性能图

Fig. 9 Electrochemical cycling performance of (a) $\mathrm{Co8555}-\mathrm{LiOH}$ and $\mathrm{Zr8555}-\mathrm{LiOH}$; (b) $\mathrm{Co8555}-\mathrm{LiOH} / \mathrm{Li}_{2} \mathrm{CO}_{3}$ and $\mathrm{Zr8555}-\mathrm{LiOH} / \mathrm{Li}_{2} \mathrm{CO}_{3}$ at $0.2 C$.

材料的电化学性能, 如图9a所示。Co8555-LiOH 的初始放电容量 $163.9 \mathrm{mAh} \cdot \mathrm{g}^{-1}$, 最高放电容量为 $169.9 \mathrm{mAh} \cdot \mathrm{g}^{-1}$ (第2周), 循环30周容量保持率为 $77.40 \%$; Zr8555-LiOH的初始放电容量 159.7 $\mathrm{mAh} \cdot \mathrm{g}^{-1}$, 循环30周容量保持率为 $79.84 \%$ 。如图 $9 \mathrm{~b}$ 所示, $\mathrm{Co} 8555-\mathrm{LiOH} / \mathrm{Li}_{2} \mathrm{CO}_{3}$ 的初始放电容量 $181.1 \mathrm{mAh} \cdot \mathrm{g}^{-1}$, 循环 80 周容量保持率为 $92.44 \%$; $\mathrm{Zr} 8555-\mathrm{LiOH} / \mathrm{Li}_{2} \mathrm{CO}_{3}$ 的初始放电容量179.9 $\mathrm{mAh} \cdot \mathrm{g}^{-1}$, 最高放电容量为 $181.2 \mathrm{mAh} \cdot \mathrm{g}^{-1}$ (第5 周), 循环 80 周容量保持率为 $96.52 \%$ 。由此可见, 对于 $\mathrm{Ni}: \mathrm{Mn}: \mathrm{Zr} / \mathrm{Co}=0.85: 0.1: 0.05$ 的高镍正极 材料, 以 $\mathrm{LiOH} \cdot \mathrm{H}_{2} \mathrm{O}$ 和 $\mathrm{Li}_{2} \mathrm{CO}_{3}$ 混合盐为锂源合成的 材料电化学性能仍优于以 $\mathrm{LiOH} \cdot \mathrm{H}_{2} \mathrm{O}$ 为锂源合成的 正极材料。

通过优化工艺合成 $\mathrm{Zr}$ 替代Co的高镍正极材料 $\mathrm{LiNi}_{0.85} \mathrm{Mn}_{0.1} \mathrm{Zr}_{0.05} \mathrm{O}_{2}$ 具有优异的电化学循环稳定 性, 且其初始放电比容量与镍钴锰高镍三元正极 材料放电容量相当。

\section{4 结论}

本文首先采用 $\mathrm{Cr}, \mathrm{Cd}$ 和 $\mathrm{Zr}$ 替代Co合成无钴高 镍正极材料, 实验发现 $\mathrm{Cr}$ 和 $\mathrm{Cd}$ 替代Co的方案不可 行, 而Zr替代Co具有一定可行性。为进一步优化 $\mathrm{Zr}$ 替代Co合成的无钴高镍正极材料的电化学性 能, 本文进一步优化了其合成工艺。首先通过碳 酸盐共沉淀法, 合成含有 $\mathrm{Ni} 、 \mathrm{Mn}$ 和 Zr 的前驱体正 极材料。然后分别 以 $\mathrm{LiOH} \cdot \mathrm{H}_{2} \mathrm{O}$ 和 $\mathrm{LiOH} \cdot \mathrm{H}_{2} \mathrm{O} / \mathrm{Li}_{2} \mathrm{CO}_{3}$ 的混合盐为锂源, 发现以混合 盐为锂源合成的正极材料锂化结晶程度高, 表面 残锂相少, 电化学性能好。再调控碳酸盐共沉淀 合成前驱体过程中的 $\mathrm{pH}$ 值, 发现当 $\mathrm{pH}=7.8$ 时
$\mathrm{Ni} 、 \mathrm{Mn}$ 和 $\mathrm{Zr}$ 的碳酸盐共沉淀成核和晶体生长的速 率达到平衡状态，此时合成的前驱体材料中过渡 金属元素可以均匀沉淀，经过高温㷽烧得到的正 极材料电化学性能好。最后, 通过验证发现该优 化合成工艺仍然适用于合成更高镍含量的无钴正 极材料。采用碳酸盐共沉淀 $(\mathrm{pH}=7.8)$ 合成前驱 体, 经过混锂(以混合锂盐为锂源)炦烧, 得到的 无钴正极材料 $\mathrm{LiNi}_{0.85} \mathrm{Mn}_{0.1} \mathrm{Zr}_{0.05} \mathrm{O}_{2}$ 层状结构好, 锂 化程度高, 表面残锂相少, 电化学性能优异。其 首周放电容量为 $179.9 \mathrm{mAh} \cdot \mathrm{g}^{-1}$, 循环 80 周容量保 持率为 $96.52 \%$, 其电化学性能略优于同等条件下 合成的 $\mathrm{LiNi}_{0.85} \mathrm{Mn}_{0.1} \mathrm{Co}_{0.05} \mathrm{O}_{2}$ 高镍正极材料。本实 验证明 $\mathrm{Zr}$ 可以作为一种替代Co的元素掺杂进入高 镍层状正极材料中, 得到的无钴高镍正极材料 $\mathrm{LiNi}_{0.85} \mathrm{Mn}_{0.1} \mathrm{Zr}_{0.05} \mathrm{O}_{2}$ 具有较好的电化学性能和较低 的成本。

\section{References}

(1) Wang, Z.; Wu, F.; Su, Y.; Bao, L.; Chen, L.; Li, N.; Chen, S. Acta Phys. -Chim. Sin. 2012, 28, 823. [王昭, 吴锋, 苏岳锋, 包丽颖, 陈来, 李宁, 陈实. 物理化学学报, 2012, 28, 823.] doi: 10.3866/PKU.WHXB201202102

(2) Tarascon, J. M.; Aamand, M. Nature 2001, 414, 359. doi: $10.1038 / 35104644$

(3) Wu, F.; Li, Q.; Chen, L.; Zhang, Q.; Wang, Z.; Lu, Y.; Bao, L.; Chen, S.; Su, Y. ACS Appl. Mater. Interfaces 2019, 11, 36751. doi: 10.1021/acsami.9b12595

(4) Zhu, G.-L.; Zhao, C.-Z.; Huang, J.-Q.; He, C.; Zhang, J.; Chen, S.; Xu, L.; Yuan, H.; Zhang, Q. Small 2019, 15, 1805389. doi: 10.1002/smll.201805389

(5) Chen, L.; Chen, S.; Hu, D.; Su, Y.; Li, W.; Wang, Z.; Bao, L.; Wu, F. 
Acta Phys. -Chim. Sin. 2014, 30, 467. [陈来, 陈实, 胡道中, 苏岳锋, 李维康, 王昭, 包丽颖, 吴锋. 物理化学学报, 2014, 30, 467.] doi: 10.3866/PKU.WHXB201312252

(6) Li, M.; Lu, J.; Chen, Z. Adv. Mater. 2018, 30, 1800561. doi: 10.1002/adma.201800561

(7) Sakti, A.; Michalek, J. J.; Fuchs, E. R. H.; Whitacre, J. F. J. Power Sources 2015, 273, 966. 10.1016/j.jpowsour.2014.09.078

(8) Kim, J.; Lee, H.; Cha, H.; Yoon, M.; Park, M.; Cho, J. Adv. Energy Mater. 2017, 8, 1702028. doi: 10.1002/aenm.201702028

(9) Bessette, S.; Paolella, A.; Kim, C.; Zhu, W.; Hovington, P.; Gauvin, R.; Zaghib, K. Sci. Rep. 2018, 8, 17575. doi: 10.1038/s41598-018-33608-3

(10) Myung, S.-T.; Maglia, F.; Park, K.-J.; Yoon, C. S.; Lamp, P.; Kim, S. J.; Sun, Y.-K. ACS Energy Lett. 2017, 2, 196. doi: 10.1021/acsenergylett.6b00594

(11) Jiang, L.; Luo, Z.; Wu, T.; Shao, L.; Sun, J.; Liu, C.; Li, G.; Cao, K.; Wang, Q. J. Electrochem. Soc. 2019, 166, A1055. doi: 10.1149/2.0661906jes

(12) Liu, W.; Oh, P.; Liu, X.; Lee, M.-J.; Cho, W.; Chae, S.; Kim, Y.; Cho, J. Angew. Chem. Int. Ed. 2015, 54, 4440. doi: $10.1002 /$ anie. 201409262

(13) Kou, J.; Wang, Z.; Bao, L.; Su, Y.; Hu, Y.; Chen, L.; Xu, S.; Chen, F.; Chen, R.; Sun, F. Acta Phys. -Chim. Sin. 2016, 32, 717. [寇建文, 王昭, 包丽颖, 苏岳锋, 胡宇, 陈来, 徐少禹, 陈芬, 陈人杰, 孙逢春. 物理化学学报, 2016, 32, 717.] doi: 10.3866/PKU.WHXB201512301

(14) Yu, H.; Qian, Y.; Otani, M.; Tang, D.; Guo, S.; Zhu, Y.; Zhou, H. Energy Environ. Sci. 2014, 7, 1068. doi: 10.1039/C3EE42398K

(15) Wu, F.; Wang, M.; Su, Y.; Chen, S. Acta Phys. -Chim. Sin. 2009, 25, 629. [吴锋, 王萌, 苏岳锋, 陈实. 物理化学学报, 2009, 25, 629.] doi: 10.3866/PKU.WHXB20090411

(16) Wu, F.; Li, Q.; Chen, L.; Lu, Y.; Su, Y.; Bao, L.; Chen, R.; Chen, S. ChemSusChem 2019, 12, 935. doi: 10.1002/cssc.201802304

(17) Kong, F.; Liang, C.; Wang, L.; Zheng, Y.; Perananthan, S.; Longo, R. C.; Ferraris, J. P.; Kim, M.; Cho, K. Adv. Energy Mater. 2018, 9 , 1802586. doi: 10.1002/aenm.201802586

(18) Kim, J.-H.; Park, K.-J.; Kim, S. J.; Yoon, C. S.; Sun, Y.-K. J. Mater. Chem. A 2019, 7, 2694. doi: 10.1039/C8TA10438G
(19) Kim, D.; Lim, J.-M.; Lim, Y.-G.; Yu, J.-S.; Park, M.-S.; Cho, M.; Cho, K. Chem. Mater. 2015, 27, 6450. doi: 10.1021/acs.chemmater.5b02697

(20) Wu, Z.; Ji, S.; Hu, Z.; Zheng, J.; Xiao, S.; Lin, Y.; Xu, K.; Amine, K.; Pan, F. ACS Appl. Mater. Interfaces 2016, 8, 15361. doi: 10.1021/acsami.6b0373

(21) Zheng, J.; Teng, G.; Xin, C.; Zhou, Z.; Liu, J.; Li, Q.; Hu, Z.; Xu, M.; Yan, S.; Yang, W.; et al. Phys. Chem. Lett. 2017, 8, 5537. doi: 10.1021/acs.jpclett.7b02498

(22) Whittingham, M. S. Chem. Rev. 2004, 104, 4271. doi: $10.1021 / \mathrm{cr} 020731 \mathrm{c}$

(23) Jian, L. F.; Zhang, M.; Yuan, H. T.; Zhao, M.; Guo, J.; Wang, W.; Zhou, X. D.; Wang, Y. M. J. Power Sources 2007, 167, 178. doi: 10.1016/j.jpowsour.2007.01.070

(24) Kim, Y. Int. J. Quantum Chem. 2019, 119, e26028. doi: org/10.1002/qua.26028

(25) He, T.; Lu, Y.; Su, Y.; Bao, L.; Tan, J.; Chen, L.; Zhang, Q.; Li, W.; Chen, S.; Wu, F. ChemSusChem 2018, 11, 1639. doi: 10.1002/cssc.201702451

(26) Han, B.; Xu, S.; Zhao, S.; Lin, G.; Feng, Y.; Chen, L.; Ivey, D. G.; Wang, P.; Wei, W. ACS Appl. Mater. Interfaces 2018, 10, 39599. doi: $10.1021 /$ acsami.8b11112

(27) Deng, S.; Lin, Z.; Li, Y.; Xue, L.; Li, H.; Chen, Y.; Lei, T.; Zhu, J.; Li, J.; Zhang, J. Int. J. Mater. Res. 2018, 109, 1043. doi: $10.3139 / 146.111701$

(28) Ma, Y.; Li, L.; Wang, L.; Luo, R.; Xu, S.; Wu, F.; Chen, R. J. Alloys Compd. 2019, 778, 643. doi: 10.1016/j.jallcom.2018.11.189

(29) Cui, Y. F.; Cui, J. L.; Man, J. Z.; Cheng, F. P.; Zhang, P. C.; Li, S.; Wen, Z. H.; Sun, J. C. Rare Metal Mat. Eng. 2019, 48, 587. [崔永福, 崔金龙, 满建宗, 程付鹏, 张鹏超, 李嵩, 文钟晟, 孙俊才. 稀有金属 材料与工程, 2019, 48, 587.]

(30) Wang, D.; Belharouak, I.; Koenig, G. M., Jr.; Zhou, G.; Amine, K. J. Mater. Chem. C 2011, 21, 9290. doi: 10.1039/C1JM11077B

(31) Wu, F.; Li, Q.; Bao, L.; Zheng, Y.; Lu, Y.; Su, Y.; Wang, J.; Chen, S.; Chen, R.; Tian, J. Electrochim. Acta 2018, 260, 986. doi: 10.1016/j.electacta.2017.12.034 